\title{
UNIQUENESS OF THE DE SITTER SPACETIME AMONG STATIC VACUA WITH POSITIVE COSMOLOGICAL CONSTANT
}

\author{
OUSSAMA HIJAZI, SEBASTIÁN MONTIEL, AND SIMON RAULOT
}

\begin{abstract}
We prove that, among all $(n+1)$-dimensional spin static vacua with positive cosmological constant, the de Sitter spacetime is characterized by the fact that its spatial Killing horizons have minimal modes for the Dirac operator. As a consequence, the de Sitter spacetime is the only vacuum of this type for which the induced metric tensor on some of its Killing horizons is at least equal to that of a round $(n-1)$-sphere. This extends uniqueness theorems shown in 1, 2, by Boucher-Gibbons-Horowitz and Chruściel to more general horizon metrics and to the non-single horizon case.
\end{abstract}

\section{INTRODUCTION}

An $(n+1)$-dimensional vacuum spacetime with cosmological constant $\Lambda$ is a Lorentzian manifold $\left(\mathcal{V}, g_{a b}\right)$ satisfying the Einstein equation $R_{a b}=\Lambda g_{a b}$, where $R_{a b}$ is the Ricci tensor of the metric $g_{a b}$. The vacuum is said to be static when

$$
\mathcal{V}=\mathbb{R} \times M, \quad d s^{2}=-V^{2} d t^{2}+{ }^{n} g_{a b} d x^{a} d x^{b},
$$

where $\left(M,{ }^{n} g_{a b}\right)$ is an $n$-dimensional connected Riemannian manifold, that we will take to be orientable, standing for the unchanging slices of constant time and $V \in C^{\infty}(M)$ is a non-trivial smooth function on $M$. In the case of positive cosmological constant $\Lambda>0$, it seems physically natural to require spatially compact solutions, that is, the Cauchy hypersurface $M$ is usually taken to be compact. The vacuum Einstein equation can be translated into the following two conditions

Date: June 22, 2018.

1991 Mathematics Subject Classification. Differential Geometry, Global Analysis, 53C27, 53C40, 53C80, 58G25.

Key words and phrases. Static vacuum, de Sitter spacetime, Killing horizon, Dirac operator.

The second author was partially supported by a Spanish MEC-FEDER grant No. MTM2011-22547. 
on $\left(M,{ }^{n} g_{a b}\right)$ and $V$ :

$$
\nabla_{a} \nabla_{b} V=V\left({ }^{n} R_{a b}-\Lambda^{n} g_{a b}\right), \quad \nabla^{2} V=-\Lambda V,
$$

where ${ }^{n} R_{a b}$ is the Ricci tensor, $\nabla_{a}$ is the covariant derivative and $\nabla^{2}=\nabla_{a} \nabla^{a}={ }^{n} g^{a b} \nabla_{a} \nabla_{b}$ is the Laplace operator of the Riemannian manifold $\left(M,{ }^{n} g_{a b}\right)$. By the second equation, we see that the spacetime cannot be globally static, that is, the lapse function $V$ changes sign on $M$. This means that the causal Killing field $\frac{\partial}{\partial t}$ is lightlike on some locus (in fact, on a hypersurface, as we will point out below) of $\mathcal{V}$. Taking traces in the first equation in (2) and taking into account the second one, we conclude immediately that

$$
{ }^{n} R=(n-1) \Lambda,
$$

where ${ }^{n} R$ is the scalar curvature of $\left(M,{ }^{n} g_{a b}\right)$.

Denote by $\Sigma \subset M$ the zero set of the lapse function $V$. If there exists a point $x \in \Sigma$ with $\left(\nabla_{a} V\right)(x)=0$, then the unicity of the solutions to the first (integrable linear) equation in (2) would imply that $V$ is identically zero on $M$. This means that $\Sigma$ is a closed smooth hypersurface embedded in $M$, although it may have several connected components. Then the null hypersurface $\mathbb{R} \times \Sigma$ of the spacetime $\mathcal{V}$ is called a Killing horizon, because it is the locus of $\mathcal{V}$ where the causal non vanishing Killing vector field $\frac{\partial}{\partial t}$ is lightlike. From a physical point of view, one usually says that $\mathbb{R} \times \Sigma$ is a cosmological event horizon, the horizon occurring because of the rapid expansion of the space due to the $\Lambda$ term. A common abuse of language will allow us to call horizon to the projected hypersurface $\Sigma$ of $M$. Indeed, each component $\Omega$ of $M$ where $V$ is positive stands for a spatial region where communication is possible and the components $\Sigma_{\alpha}, \alpha=1, \ldots, k$, of its boundary $\partial \Omega=\Sigma$ represent unattainable barriers for signals. From now on, we will restrict ourselves to work on open domains $\Omega \subset M$ of this type with compact closure $\bar{\Omega}$ and non empty boundary $\Sigma=\partial \Omega$, not necessarily connected. Since the Riemannian manifold with non empty boundary $\left(\Omega,{ }^{n} g_{a b}\right)$, with ${ }^{n} g_{a b} \in C^{\infty}(\bar{\Omega})$ and $V \in C^{\infty}(\bar{\Omega})$, positive on $\Omega$ and vanishing along $\Sigma$, completely determine the physically realistic regions of the vacuum spacetime $\left(\mathcal{V}, g_{a b}\right)$. It is also usual to call the triple $\left(\Omega,{ }^{n} g_{a b}, V\right)$ a positive static triple.

The paradigmatic example of a positive static triple with cosmological constant $\Lambda>0$ is given by choosing $\left(\Omega,{ }^{n} g_{a b}\right)=\left(\mathbb{S}_{+}^{n}\left(\sqrt{\frac{n}{\Lambda}}\right),{ }^{n} \delta_{a b}\right)$ the open upper $n$-hemisphere in $\mathbb{R}^{n+1}$ of radius $\sqrt{\frac{n}{\Lambda}}$, where ${ }^{n} \delta_{a b}$ is the Euclidean metric tensor. In particular, ${ }^{n} R_{a b}=\frac{\Lambda}{n}(n-1)^{n} \delta_{a b}$ and 
$\Sigma=\partial \Omega=\mathbb{S}^{n-1}\left(\sqrt{\frac{n}{\Lambda}}\right)$ is the equator. It is easy to see that, if $\mathbf{p}$ is the pole of $\mathbb{S}_{+}^{n}\left(\sqrt{\frac{n}{\Lambda}}\right)$, the corresponding height function $h$ given by

$$
h(x)=x^{a} \mathbf{p}_{a}, \quad \forall x \in \mathbb{S}_{+}^{n}\left(\sqrt{\frac{n}{\Lambda}}\right)
$$

is positive on $\mathbb{S}_{+}^{n}\left(\sqrt{\frac{n}{\Lambda}}\right)$, vanishes along $\Sigma=\mathbb{S}^{n-1}\left(\sqrt{\frac{n}{\Lambda}}\right)$ and satisfies the Obata type equation (see [3, 4])

$$
\nabla_{a} \nabla_{b} h=-\frac{\Lambda}{n} h^{n} \delta_{a b}
$$

As a consequence, $\nabla^{2} h=-\Lambda h$, and so equations (2) are satisfied. Indeed, the corresponding spacetime

$$
\left(d S_{+}, d s^{2}\right)=\left(\mathbb{R} \times \mathbb{S}_{+}^{n},-h^{2} d t^{2}+{ }^{n} \delta_{a b} d x^{a} d x^{b}\right)
$$

is nothing but an open domain in de Sitter spacetime of radius $\sqrt{\frac{n}{\Lambda}}$ bounded by a certain lightlike hypersurface.

In fact, the so called cosmic no-hair conjecture, formulated by BoucherGibbons-Horowitz (see [1, p. 2449]) refers to a postulated and desired uniqueness for the above example:

The only $(n+1)$-dimensional static vacuum spacetime with $\Lambda>0$ and connected cosmic event horizon is the de Sitter spacetime of radius $\sqrt{\frac{n}{\Lambda}}$. In other words, the only $n$-dimensional positive static triple $\left(\Omega,{ }^{n} g_{a b}, V\right)$ with single-horizon $\Sigma=\partial \Omega$ and cosmological constant $\Lambda>0$ is given by a round hemisphere $\left(\mathbb{S}_{+}^{n}\left(\sqrt{\frac{n}{\Lambda}}\right),{ }^{n} \delta_{a b}\right)$ of radius $\sqrt{\frac{n}{\Lambda}}$, where the lapse function $V$ is taken as the height function attaining its maximum at the pole.

Connectedness of the horizon $\Sigma$ is essential for this conjecture to be true. In fact, we can easily construct positive vacuum triples $\left(\Omega,{ }^{n} g_{a b}, V\right)$ with double-horizon $\Sigma$. It suffices to take $\Omega=\mathbb{S}_{+}^{1}\left(\frac{1}{\sqrt{\Lambda}}\right) \times P$, where $\mathbb{S}_{+}^{1}\left(\frac{1}{\sqrt{\Lambda}}\right)$ is the upper half-circle of radius $\frac{1}{\sqrt{\Lambda}}$ and $P$ is any $(n-1)$-dimensional Einstein compact manifold with Ricci curvature equal to $\Lambda$ (for example, the choice of $P$ as the sphere $\mathbb{S}^{n-1}\left(\sqrt{\frac{n-2}{\Lambda}}\right)$ provides the so called Nariai spacetime), ${ }^{n} g_{a b}$ the product metric and $V(t, x)=\sin \sqrt{\Lambda} t$ for all $\frac{1}{\sqrt{\Lambda}} e^{i \sqrt{\Lambda} t} \in \mathbb{S}_{+}^{1}\left(\frac{1}{\sqrt{\Lambda}}\right)$ and all $x \in P$. One can check (see, for example, [5, p. 51, 2.]) that these choices ensure that equations (2) are satisfied. Moreover, it is immediate that, in this case, $\Sigma$ consists just of two copies of $P$. 
The cosmic no-hair conjecture is closely related to another conjecture by Fischer and Marsden concerning the Riemannian metrics which are critical points for the scalar curvature map (see [6, 7, 8]). Indeed, we may rephrase this Fischer-Marsden conjecture (see [9, Conjecture 2.]) just as the Boucher-Gibbons-Horowitz conjecture above by removing the single-horizon assumption. So, the last aforementioned examples should be viewed as counterexamples of this conjecture, at least as long as stated in such a generality.

Since the gradual spreading of the cosmic no-hair conjecture, some results about the subject have been interpreted as advances lending support to it. Firstly, it is clear from (2) that, if $\left(\Omega,{ }^{n} g_{a b}\right)$ is Einstein (or even if just the Ricci tensor ${ }^{n} R_{a b}$ has $\Lambda$ as a lower or upper bound), the Obata type theorem in [10] (cf. [4]) solves the conjecture in the affirmative, even in the case where the horizon $\Sigma$ is not assumed in principle to be connected. The same positive answer is obtained when $\left(\Omega,{ }^{n} g_{a b}\right)$ is supposed to be conformally flat, a result proved independently by Kobayashi in [11] and Lafontaine in [12]. Moreover, Chruściel, generalizing some early computations by Lindblom in [13], showed in [2] the following integral inequality

$$
\sum_{\alpha=1}^{k} \kappa_{\alpha} \int_{\Sigma_{\alpha}}\left({ }^{(n-1)} R_{\alpha}-\frac{\Lambda}{n}(n-1)(n-2)\right) d \Sigma_{\alpha} \geq 0
$$

where ${ }^{(n-1)} R_{\alpha}$ is the scalar curvature of the metric tensor ${ }^{(n-1)} h_{i j}^{\alpha}$ induced from $\Omega$ on the connected component $\Sigma_{\alpha}$ of the event horizon $\Sigma$ and each constant $\kappa_{\alpha}>0$ is the corresponding surface gravity on $\Sigma_{\alpha}$ (see (8) for a definition). The equality implies that $\Omega$ is the round hemisphere of radius $\sqrt{\frac{n}{\Lambda}}$ (and we have $k=1$ a fortiori, that is, $\Sigma$ must be connected).

Inequality (4) has two important consequences. Clearly, it implies that at least one of the integrals in the sum must be non negative. Since an $(n-1)$-dimensional round sphere of radius $\sqrt{\frac{n}{\Lambda}}$ has constant scalar curvature $\frac{\Lambda}{n}(n-1)(n-2)$, at least one of the components $\Sigma_{\alpha}$ of the event horizon $\Sigma$ has total scalar curvature greater than or equal to that of such a sphere. In the single-horizon case, this means that:

The de Sitter vacuum minimizes the integral of the scalar curvature of the induced metric on the event horizon among all the single-horizon positive static triples with the same cosmological constant. 
In the case $n=3$, inequality (4) along with the considerations above imply that the horizon has at least one genus zero component and, in the single-horizon case, one obtains the inequality

$$
12 \pi \geq \Lambda \text { area }(\Sigma)
$$

discovered by Boucher-Gibbons-Horowitz in [1] (see also [9] for an analogous computation in the case of multiple horizons). As a consequence of these last inequalities, one has the following uniqueness result by Chruściel (see [2, 9]):

Let $\left(\Omega,{ }^{n} g_{a b}, V\right)$ be an $n$-dimensional positive static triple with cosmological constant $\Lambda>0$ and suppose that the horizon $\left(\Sigma,{ }^{(n-1)} h_{i j}\right)$ is isometric to a sphere $\mathbb{S}^{n-1}(r)$ of radius $r>0$. Then $r \leq \sqrt{\frac{n}{\Lambda}}$, and if the equality holds, the triple $\left(\Omega,{ }^{n} g_{a b}, V\right)$ is given by a round hemisphere $\left(\mathbb{S}_{+}^{n}\left(\sqrt{\frac{n}{\Lambda}}\right),{ }^{n} \delta_{a b}\right)$ of radius $r=\sqrt{\frac{n}{\Lambda}}$, where the lapse function $V$ is a height function attaining its maximum at the pole.

In fact, in order to have the uniqueness result above, it would be enough that the scalar curvature ${ }^{(n-1)} R$ of $\left(\Sigma,{ }^{(n-1)} h_{i j}\right)$ is at most equal to that of the sphere $\mathbb{S}^{n-1}\left(\sqrt{\frac{n}{\Lambda}}\right)$, that is, ${ }^{(n-1)} R \leq \frac{(n-1)(n-2)}{n} \Lambda$.

Eventhough all these results have been thought of as evidences for solving affirmatively the cosmic no-hair conjecture, at least in the single-horizon case, they should be viewed as signs that the desired uniqueness of the de Sitter spacetime seems to require some boundary condition, that is, some topological, geometrical or analytical assumptions on the cosmic event horizon, as in the case of negative cosmological constant $\Lambda$ (see [1, 14, 15, 16, 17, 18, 19, 20, 21, 22, ) or zero (see [23, 24, 25, 26]). This point of view has been confirmed by GibbonsHartnoll-Pope, who constructed in [27] counterexamples to the cosmic no-hair conjecture, in the cases $4 \leq n \leq 8$, by using the Riemannian inhomogeneous Einstein metrics found by Böhm in [28] (as well as by the uniqueness resuts obtained in [1, 2, 29, 30]). In these counterexamples, one can find event horizons which are topologically spherical but endowed with non round metrics and Riemannian products of spheres. Anyway, Gibbons-Hartnoll-Pope showed that these examples are dinamically unstable and should evolve into an asymptotically de Sitter spacetime.

Therefore, taking into account the remarks above and the uniqueness results obtained by Chruściel in 2] and by Boucher-Gibbons-Horowitz 
in [1, it becomes interesting to find necessary conditions, like inequalities (4) and (5), on a given $(n-1)$-dimensional compact Riemannian manifold $\left(\Sigma,{ }^{(n-1)} h_{i j}\right)$ in order to be a connected component of the event horizon of a positive static triple $\left(\Omega,{ }^{n} g_{a b}, V\right)$ with cosmological constant $\Lambda>0$. In this way, we will be able to approach to the uniqueness of the Sitter spacetime imposing natural conditions on the event horizon and ruling out the single-horizon assumption.

Indeed, in this paper, in the first place we will prove that the de Sitter spacetime minimizes the absolute value of the modes of the Dirac operator on each component of the event horizon among all the positive static triples. Precisely, we will show the following result.

Theorem 1. Let $\left(\Omega,{ }^{n} g_{a b}, V\right)$ be a positive static triple with cosmological constant $\Lambda>0$. Suppose that $\Omega$ is a spin manifold (this is always the case if $n=3$ ) and that $\partial_{\alpha}$ and $\kappa_{\alpha}$ are respectively the Dirac operator of the Riemannian spin structure induced on the connected component $\Sigma_{\alpha}, \alpha=1, \ldots, k$, of the event horizon $\Sigma=\partial \Omega$ and the surface gravity of $\Sigma_{\alpha}$. Then

$$
\left|\lambda\left(\partial_{\alpha}\right)\right| \geq \frac{n-1}{2} \sqrt{\frac{\Lambda}{n}}\left(\frac{\kappa_{\alpha}}{\kappa_{\max }}\right),
$$

for all the modes $\lambda$ of $\not_{\alpha}$, where $\kappa_{\max }=\max _{\alpha=1, \ldots, k} \kappa_{\alpha}$. If equality holds for some $\alpha=1, \ldots, k$, then $k=1$, that is, the event horizon $\Sigma$ is connected, $\kappa_{\alpha}=\kappa_{\max }$, the Riemannian manifold $\left(\Omega,{ }^{n} g_{a b}\right)$ is given by a round hemisphere $\left(\mathbb{S}_{+}^{n}\left(\sqrt{\frac{n}{\Lambda}}\right),{ }^{n} \delta_{a b}\right)$ of radius $\sqrt{\frac{n}{\Lambda}}$ and the lapse function $V$ is a height function attaining its maximum at the pole.

Note that the lower bound obtained in Theorem 1 is independent of scale changes in the lapse function $V$. In fact, it is obvious from (2) that, if $V$ is a lapse function, any multiple of $V$ is a lapse function as well.

As a consequence, and by using a nice upper estimate of the Dirac operator on spheres due to Herzlich (see [31]), who improved an original general estimate by Vafa-Witten (see [32]), we obtain another result which generalises the Chruściel and Boucher-Gibbons-Horowitz theorem in two directions: it applies to the general case of non singlehorizon $\Sigma$ and moreover there is no need to impose that the metric tensor on the horizon is the round spherical metric, but only that it dominates this round metric.

Theorem 2. Let $\left(\Omega,{ }^{n} g_{a b}, V\right)$ be an $n$-dimensional positive static triple with cosmological constant $\Lambda>0$ and suppose that $\Omega$ is spin and that 
there is a connected component $\Sigma_{0}$ of the event horizon $\Sigma=\partial \Omega$ which is diffeomorphic to an $(n-1)$-dimensional sphere. If the corresponding induced tensor metric ${ }^{(n-1)} h_{i j}^{0}$ is pointwise at least equal to that of the round standard sphere of radius $r>0$, then $r \leq \sqrt{\frac{n}{\Lambda}}\left(\frac{\kappa_{\max }}{\kappa_{0}}\right)$. If equality is attained, then $\Sigma$ is connected, $\kappa_{0}=\kappa_{\max }$, the Riemannian manifold $\left(\Omega,{ }^{n} g_{a b}\right)$ is given by a round hemisphere $\left(\mathbb{S}_{+}^{n}\left(\sqrt{\frac{n}{\Lambda}}\right),{ }^{n} \delta_{a b}\right)$ of

radius $r=\sqrt{\frac{n}{\Lambda}}$ and the lapse function $V$ is a height function attaining its maximum at the pole.

\section{Geometry of a modification of the Fermat conformal METRIC}

Given a positive static triple $\left(\Omega,{ }^{n} g_{a b}, V\right)$, the conformal metric $\widetilde{n}_{a b}=\frac{1}{V^{2}} n_{a b}$ on $\Omega$ is known as the Fermat (or optical) metric. This is why the geodesics of this new metric are the spatial projections of the light rays in the corresponding spacetime $\left(\mathbb{R} \times \Omega,-V^{2} d t^{2}+\right.$ $\left.{ }^{n} g_{a b} d x^{a} d x^{b}\right)$. The geometrical features of this conformal metric have been explicitly or implicitly studied in order to analyse the behaviour of static spacetimes, mainly with non-null cosmological constant (see, for example, [1, 17, 18, 13, 33]). In [2, 19], a suitable modification of this metric has been used. We will consider again this modified Fermat metric in order to prove Theorems 1 and 2.

Lemma 3. Let $\left(\Omega,{ }^{n} g_{a b}, V\right)$ be a positive static vacuum and $\varepsilon>0$ an arbitrary positive real number. Then the conformal Riemannian metric ${ }^{n} g_{a b}^{*}=\left(\frac{\varepsilon}{V+\varepsilon}\right)^{2}{ }^{n} g_{a b}$ has scalar curvature

$$
{ }^{n} R^{*}=\frac{(n-1) \Lambda}{\varepsilon^{2}}\left(\varepsilon^{2}-V^{2}-\frac{n}{\Lambda} W\right),
$$

where $W$ is the squared norm of the gradient of the lapse function $V$, that is, $W={ }^{n} g^{a b} \nabla_{a} V \nabla_{b} V$. Moreover, ${ }^{n} g_{a b}^{*}$ coincides with ${ }^{n} g_{a b}$ on the event horizon $\Sigma=\partial \Omega$.

Proof. The last assertion is clear because $V$ vanishes along $\Sigma$. If we rewrite the conformal change between the metrics ${ }^{n} g_{a b}$ and ${ }^{n} g_{a b}^{*}$ as

$$
{ }^{n} g_{a b}=\left(\frac{V+\varepsilon}{\varepsilon}\right)^{2}{ }^{n} g_{a b}^{*}
$$

then the relation between the corresponding Ricci tensors ${ }^{n} R_{a b}^{*}$ and ${ }^{n} R_{a b}$ on the compact manifold $\bar{\Omega}$, is given by (see [34, p. 59]):

$$
{ }^{n} R_{a b}^{*}={ }^{n} R_{a b}+(n-2) \frac{\nabla_{a} \nabla_{b} V}{V+\varepsilon}+\frac{\nabla^{2} V}{V+\varepsilon}{ }^{n} g_{a b}-(n-1) \frac{W}{(V+\varepsilon)^{2}}{ }^{n} g_{a b} .
$$


Taking traces with respect to ${ }^{n} g_{a b}$ and multiplying by $(V+\varepsilon)^{2}$, we obtain the corresponding relation for their scalar curvatures

$$
\varepsilon^{2}\left({ }^{n} R^{*}\right)={ }^{n} R(V+\varepsilon)^{2}+2(n-1)(V+\varepsilon) \nabla^{2} V-n(n-1) W .
$$

As we pointed out in (2) and (3) , since ${ }^{n} R=(n-1) \Lambda$ and the function $V$ is an eigenfunction of $\nabla^{2}$ associated with the eigenvalue $-\Lambda$, we finally get the required expression for the scalar curvature ${ }^{n} R^{*}$ of the compact Riemannian manifold with boundary $\left(\bar{\Omega},{ }^{n} g_{a b}^{*}\right)$ q.e.d.

Our aim now is to prove that the conformal metric ${ }^{n} g_{a b}^{*}$ has nonnegative scalar curvature ${ }^{n} R^{*}$. The proof of this assertion will be a reformulation of an identity due to Lindblom ([13]) showing that ${ }^{n} R^{*}$ satisfies a certain elliptic second order equation on $\bar{\Omega}$ and a short analysis displaying the geometry of the event horizon $\Sigma$ as a hypersurface of $\bar{\Omega}$. Let us start by this last point. In fact, in the discussion after equality (3), we noticed that along the event horizon $\Sigma$, which is the zero set of the lapse function $V$, the gradient vector field $\nabla^{a} V$ has no zeroes and so, after normalization, it provides an inner normal vector field $N^{a}$ for the hypersurface $\Sigma$ in $\bar{\Omega}$. Then, it is well-known that the extrinsic curvature (or second fundamental form) $p_{i j}=-{ }^{n} g_{i c} \nabla_{j} N^{c}$ of any regular level set of $V$ is given by the equation

$$
\nabla_{i} \nabla_{j} V+\left(N^{c} \nabla_{c} V\right) p_{i j}=0 .
$$

Since $N^{c} \nabla_{c} V$ has no zeroes on $\Sigma$ (which is the regular level set $V^{-1}(\{0\})$ ), by the first equality in (2), we have

$$
p_{i j}=0 \text { on } \Sigma \text {, that is, } \Sigma \text { is totally geodesic in } \bar{\Omega} \text {. }
$$

Then, the fact that $p_{i j}=0$ and (2) give

$$
\nabla_{i}\left(N^{b} \nabla_{b} V\right)_{\mid \Sigma}=-{ }^{n} g^{b j} p_{i j} \nabla_{b} V+N^{b}\left(\nabla_{i} \nabla_{b} V\right)_{\mid \Sigma}=0
$$

and so the normal derivative $N^{a} \nabla_{a} V$ is constant on each connected component of the event horizon $\Sigma$. Recall that $\Sigma_{\alpha}, \alpha=1, \ldots, k$, denote its connected components. So, we dzfinz

$$
\kappa_{\alpha} \equiv\left(N^{a} \nabla_{a} V\right)_{\mid \Sigma_{\alpha}}>0, \quad \alpha=1, \ldots, k .
$$

Note that each $\kappa_{\alpha}$ is positive because, as we pointed out in the discussion following equality (3), the gradient of $V$ never vanishes on its zero set. Physically, each $\kappa_{\alpha}$ is the surface gravity on the component $\Sigma_{\alpha}$ of the event horizon of the $n$-dimensional spatial slice $\Omega$.

Proposition 4. Let $\left(\Omega,{ }^{n} g_{a b}, V\right)$ be an $n$-dimensional positive static triple with cosmological constant $\Lambda>0$ and let $\Sigma_{\alpha}, \alpha=1, \ldots, k$, be the connected components of its event horizon. Then, taking 
$\varepsilon=\sqrt{\frac{n}{\Lambda}} \kappa_{\max }>0$, where $\kappa_{\max }$ is the greatest surface gravity among all the components $\Sigma_{\alpha}$, yields

$$
{ }^{(n-1)} h_{i j}^{*}={ }^{(n-1)} h_{i j}, \quad{ }^{n} R^{*} \geq 0, \quad\left(p^{*}\right)_{\mid \Sigma_{\alpha}}=(n-1) \sqrt{\frac{\Lambda}{n}}\left(\frac{\kappa_{\alpha}}{\kappa_{\max }}\right),
$$

where ${ }^{n} R^{*}$ is the scalar curvature of the conformal metric ${ }^{n} g_{a b}^{*}$ defined in Lemma 3, $p^{*}$ is the trace of the (inner) extrinsic curvature $p_{i j}^{*}$ of the event horizon $\Sigma=\partial \Omega$ as a hypersurface of $\left(\bar{\Omega},{ }^{n} g_{a b}^{*}\right)$.

Proof. The first equality is simply the last assertion in the statement of Lemma 3. As for the second one, we define a function $\Phi$ on $\bar{\Omega}$ by

$$
\Phi=\varepsilon^{2}-V^{2}-\frac{n}{\Lambda} W=\varepsilon^{2}-V^{2}-\frac{n}{\Lambda}{ }^{n} g^{a b} \nabla_{a} V \nabla_{b} V
$$

that is, up to a positive constant, the righthand side of (6). Taking into account the more or less explicit computations in [13, 2, 33] or the classical Bochner formula for the Laplace operator of the squared length of a gradient, we have

$$
\begin{aligned}
\frac{1}{2} \nabla^{2} \Phi & =-W-V \nabla^{2} V-\frac{n}{\Lambda}\left(\nabla_{a} \nabla_{b} V\right)\left(\nabla^{a} \nabla^{b} V\right) \\
& -\frac{n}{\Lambda} \nabla_{a} V \nabla^{a}\left(\nabla^{2} V\right)-\frac{n}{\Lambda}{ }^{n} R_{a b} \nabla^{a} V \nabla^{b} V .
\end{aligned}
$$

Using (2), we obtain

$$
\begin{aligned}
\frac{1}{2} \nabla^{2} \Phi & =-\frac{n}{\Lambda}\left(\nabla_{a} \nabla_{b} V+\frac{\Lambda}{n} V^{n} g_{a b}\right)\left(\nabla^{a} \nabla^{b} V+\frac{\Lambda}{n} V^{n} g^{a b}\right) \\
& -\frac{n}{\Lambda} \frac{\left(\nabla_{a} \nabla_{b} V\right)\left(\nabla^{a} V \nabla^{b} V\right)}{V}-W .
\end{aligned}
$$

On the other hand, from the definition of $\Phi$, we have

$$
\nabla_{a} \Phi \nabla^{a} V=-2 V W-2 \frac{n}{\Lambda} \nabla_{a} \nabla_{b} V \nabla^{a} V \nabla^{b} V .
$$

Hence, by combining (9) and (10), we finally obtain

$$
\begin{aligned}
\frac{1}{2} \nabla^{2} \Phi & =-\frac{n}{\Lambda}\left(\nabla_{a} \nabla_{b} V+\frac{\Lambda}{n} V^{n} g_{a b}\right)\left(\nabla^{a} \nabla^{b} V+\frac{\Lambda}{n} V^{n} g^{a b}\right) \\
& +\frac{1}{2 V} \nabla_{a} \Phi \nabla^{a} V .
\end{aligned}
$$

A standard application of min-max principle to this elliptic equation (11) implies that $\Phi$ reaches its minimum value at $\Sigma$. Then, to control the behaviour of the function $\Phi$ along the event horizon, it suffices to recall that $\Sigma=V^{-1}(\{0\})$ and that

$$
W_{\mid \Sigma}=\left(\nabla_{a} V \nabla^{a} V\right)_{\mid \Sigma}=\left(N^{a} \nabla_{a} V\right)^{2} \leq \frac{\Lambda}{n} \varepsilon^{2},
$$


according to (8) and the choice of $\varepsilon$. Therefore, we conclude that

$$
\Phi_{\mid \Sigma}=\left(\varepsilon^{2}-V^{2}-\frac{n}{\Lambda} W\right)_{\mid \Sigma} \geq 0
$$

and that, if $\Phi$ is not constant, the equality holds only on the connected components of $\Sigma$ with maximum surface gravity. This proves that $\Phi \geq 0$ on the whole of $\Omega$ and so, using (마) in Lemma 3 , we obtain the second assertion ${ }^{n} R^{*} \geq 0$.

To finish the proof, it remains to compute the mean curvature $p^{*}$ of the event horizon $\Sigma$ as a hypersurface of the compact Riemannian manifold $\left(\bar{\Omega},{ }^{n} g_{a b}^{*}\right)$. Observe that, by definition in Lemma 3 .

$$
{ }^{n} g_{a b}^{*}=\left(\frac{\varepsilon}{V+\varepsilon}\right)^{2}{ }^{n} g_{a b} .
$$

But, we know from (17) that the extrinsic curvature $p_{a b}$ of the event horizon $\Sigma$ as a hypersurface of $\left(\bar{\Omega},{ }^{n} g_{a b}\right)$ vanishes (in fact, $\Sigma$ is a totally geodesic hypersurface). So, in order to compute $p^{*}$, it suffices to use the well-known relation between the two mean curvatures of a hypersurface corresponding to two metrics on the ambient space which are conformal (see, for instance, [33]):

$$
p^{*}=\frac{1}{\rho}\left(p-(n-1) N^{a} \nabla_{a} \log \rho\right)=-\frac{n-1}{\rho^{2}} N^{a} \nabla_{a} \rho,
$$

where $\rho=\frac{\varepsilon}{V+\varepsilon}$ and $N^{a}$ is the inner unit normal along $\Sigma$ with respect to the metric ${ }^{n} g_{a b}$. Since we have

$$
\left(\nabla_{a} \rho\right)_{\mid \Sigma}=-\left.\frac{\varepsilon \nabla_{a} V}{(V+\varepsilon)^{2}}\right|_{\Sigma}=-\frac{\rho^{2}}{\varepsilon}\left(\nabla_{a} V\right)_{\mid \Sigma} .
$$

Thus, we obtain

$$
p^{*}=\frac{n-1}{\varepsilon}\left(N^{a} \nabla_{a} V\right)_{\mid \Sigma} .
$$

Taking into account (8) and the choice of $\varepsilon$, for any $\alpha=1, \ldots, k$, we finally have

$$
\left(p^{*}\right)_{\mid \Sigma_{\alpha}}=\frac{n-1}{\varepsilon} \kappa_{\alpha}=(n-1) \sqrt{\frac{\Lambda}{n}}\left(\frac{\kappa_{\alpha}}{\kappa_{\max }}\right) .
$$

q.e.d.

Remark 1. Note that equation (11) is equivalent to

$$
\frac{1}{2} \operatorname{div} \frac{1}{V} \nabla \Phi=-\frac{n}{\Lambda}\left(\nabla_{a} \nabla_{b} V+\frac{\Lambda}{n} V^{n} g_{a b}\right)\left(\nabla^{a} \nabla^{b} V+\frac{\Lambda}{n} V^{n} g^{a b}\right),
$$


where it can easily checked that the vector field $\frac{1}{V} \nabla \Phi$ extends smoothly to the boundary $\Sigma$. So, we have the inequality

$$
\operatorname{div} \frac{1}{V} \nabla \Phi \leq 0
$$

By integrating and using the divergence theorem, we obtain

$$
\int_{\Sigma}\left(\frac{1}{V} N^{a} \nabla_{a} \Phi\right) d \Sigma \geq 0
$$

where $N=\frac{\nabla V}{|\nabla V|}$ is the inner unit normal along $\Sigma$. Now, by using the definitions of $\Phi$ and of the $\kappa_{\alpha}, \alpha=1, \ldots, k$, we have that

$$
\left(\frac{1}{V} N^{a} \nabla_{a} \Phi\right)_{\mid \Sigma_{\alpha}}=\kappa_{\alpha}\left(\frac{n}{\Lambda}{ }^{n} R_{a b} N^{a} N^{b}-(n-1)\right) .
$$

Then, equations (44) and (5) are direct consequences from the discussion above and the Gauß equation

$$
{ }^{(n-1)} R=(n-1) \Lambda-2{ }^{n} R_{a b} N^{a} N^{b}
$$

on the totally geodesic hypersurface $\Sigma$.

\section{Proofs of Theorems 1 and 2}

Suppose now that the positive static triple $\left(\Omega,{ }^{n} g_{a b}, V\right)$ is such that the compact orientable $n$-dimensional manifold $\bar{\Omega}$ with non empty boundary is a spin manifold (this is always the case when the spatial slice $M$ of the spacetime is spin) and that we have fixed a spin structure on it. Since the horizon $\Sigma=\partial \Omega$ is always an orientable hypersurface, it is also a spin manifold and that an induced spin structure on the horizon is inherited from the structure fixed on $\bar{\Omega}$. Moreover, for the Riemanian metric ${ }^{n} g_{a b}$ on $\bar{\Omega}$ we have an associated spinor bundle $\left(\mathbb{S} \bar{\Omega}, \gamma^{a}, \bar{\nabla}_{a}, \bar{\partial}\right)$, where $\gamma^{a}$ are the Pauli matrices, $\bar{\nabla}_{a}$ the covariant derivative and $\bar{\partial}$ is the corresponding Dirac operator (for generalities on spin structures see any of [36, 37, 38, 39]). It is a well-known fact that the restriction of the spinor bundle $\mathbb{S} \bar{\Omega}$ to the hypersurface $\Sigma$ can be identified with one or two copies of the spinor bundle corresponding to the induced spin structure and the induced Riemannian metric ${ }^{(n-1)} h_{i j}$ according to the parity of the dimension $n$ of $\bar{\Omega}$. More precisely, we have an isomorphism

$$
\left(\mathbb{S} \bar{\Omega}_{\mid \Sigma}, \gamma^{n} \bar{\partial}+N^{a} \bar{\nabla}_{a}-\frac{1}{2} p\right) \cong\left\{\begin{array}{l}
(\mathbb{S} \Sigma, \not \partial), \text { if } n \text { is odd } \\
(\mathbb{S} \Sigma, \not \partial) \oplus(\mathbb{S} \Sigma,-\not \partial), \text { if } n \text { is even }
\end{array}\right.
$$

where $N$ is the inner unit normal field along the horizon $\Sigma, p$ is the trace of its extrinsic curvature and $(\mathbb{S} \Sigma, \not \partial)$ are respectively the spinor 
bundle and the Dirac operator corresponding to the spin structure and to the Riemannian metric induced on $\Sigma$ (for this relationship between the spinor bundles on a hypersurface and on its ambient space, see, for instance, [40, 37, 41, 42]). Due to this identification we can say that each spinor field on $\bar{\Omega}$ determines by restriction a spinor field on the event horizon $\Sigma$ and we can talk about possible extensions to $\bar{\Omega}$ of the spinor fields defined on $\Sigma$. Furthermore, from the identification between the operators $\not \partial$ and $\gamma^{n} \bar{\partial}+N^{a} \bar{\nabla}_{a}-\frac{1}{2} p$, it is immediate that, if $p$ is constant, the restriction to $\Sigma$ of a parallel spinor field on $\bar{\Omega}$ gives an eigenspinor on $\Sigma$ associated to the eigenvalue $-\frac{1}{2} p$ of the operator $\not$. Taking into account the identifications above between bundles and operators, X. Zhang and the first two authors showed in [41] (see also [43. Theorem 3.7.1]) that, if the scalar curvature ${ }^{n} R$ of the metric ${ }^{n} g_{a b}$ is non negative on $\bar{\Omega}$ and the trace $p$ of the extrinsic curvature of $\Sigma$ in $\bar{\Omega}$ is also non negative, we have

$$
\left|\lambda_{1}(\not \partial)\right| \geq \frac{1}{2} \inf _{\Sigma} p
$$

where $\lambda_{1}(\not \partial)$ stands for the eigenvalue of $\not \partial$ with the lowest absolute value, and, if the equality holds, then the eigenspace associated to $\lambda_{1}(\not \partial)$ is built from parallel spinor fields on $\bar{\Omega}$ (note that in [41, 43] the inequality is given in terms of the normalized mean curvature of $\Sigma$ ). It is straightforward to check that the approach in [41] can be applied to each connected component $\Sigma_{\alpha}, \alpha=1, \ldots, k$, of $\Sigma$ in the case where $\Sigma$ is not connected. From equations (3) and (7), one can see that the estimate (12) can be applied to our situation. But, unfortunately, we get on each component, the obvious inequality $\left|\lambda_{1}\left(\not_{\alpha}\right)\right| \geq 0$. In fact, it is clear that (12) is of interest only when $\inf _{\Sigma} p>0$. Nevertheless, we can obtain some significant information by considering the conformal metric ${ }^{n} g_{a b}^{*}$ on $\bar{\Omega}$ (see Lemma 3 and Proposition 4). By combining the information provided by Proposition 4 and estimate (12), we get

$$
\left|\lambda_{1}\left(\not \partial_{\alpha}\right)\right| \geq \frac{n-1}{2} \sqrt{\frac{\Lambda}{n}}\left(\frac{\kappa_{\alpha}}{\kappa_{\max }}\right),
$$

for each $\alpha=1, \ldots, k$, as required in Theorem 11. As we mentioned before, according to [41, if the equality holds, then the eigenspace associated with $\lambda_{1}(\not \partial)$ is built of parallel spinor fields on $\left(\bar{\Omega},{ }^{n} g_{a b}^{*}\right)$. This implies the existence of a non trivial parallel spinor field $\Psi \in \Gamma \mathbb{S} \bar{\Omega}$ with respect to the metric ${ }^{n} g_{a b}^{*}$. It was shown by Hitchin in [44] (see also [36, Chapter 6]) that the existence of a non-trivial parallel spinor forces the Ricci tensor to vanish everywhere. Then ${ }^{n} R_{a b}^{*}=0$ on $\bar{\Omega}$ and so ${ }^{n} R^{*}=0$ 
as well. From (6) and (11), we conclude

$$
\nabla_{a} \nabla_{b} V+\frac{\Lambda}{n} V^{n} g_{a b}=0 .
$$

Hence the compact Riemannian manifold with non empty boundary $\left(\bar{\Omega},{ }^{n} g_{a b}\right)$ admits a non trivial solution $V$ to the Obata equation which is positive on $\Omega$ and vanishes on its boundary $\Sigma$. Now, we may apply the non empty boundary version of the Obata theorem due to Reilly (see [10]) and conclude that $\left(\bar{\Omega},{ }^{n} g_{a b}\right)$ is a round hemisphere with radius $\sqrt{\frac{n}{\Lambda}}$ and $V$ is the height function with maximum at its center. This finishes the proof of Theorem 11.

As for Theorem 2, we suppose that there is a component $\Sigma_{0}$ of the event horizon $\Sigma$ which is diffeomorphic to a sphere $\mathbb{S}^{n-1}$. Then the simple connectedness of this component implies that it supports only one spin structure and so the spin structure induced from $\bar{\Omega}$ on $\Sigma_{0}$ is the standard one on the $(n-1)$-dimensional sphere. On the other hand, we assume that the metric ${ }^{(n-1)} h_{i j}^{0}$ induced on $\Sigma$, after applying a diffeomorphism if necessary, satisfies

$$
{ }^{(n-1)} h_{i j}^{0} \geq{ }^{(n-1)} \delta_{i j}, \quad \text { pointwise, }
$$

where ${ }^{(n-1)} \delta_{i j}$ is the round metric of radius, say, $r>0$. This hypothesis allows us to apply [31, Theorem 1] and conclude that

$$
\left|\lambda_{1}\left(\not_{0}\right)\right| \leq \frac{n-1}{2 r}
$$

where the equality implies that ${ }^{(n-1)} h_{i j}^{0}$ is just the round metric. Combining this upper bound for the eigenvalue of the Dirac operator $\not_{0}$ of the horizon with the least absolute value with the lower bound provided by Theorem 1, we have

$$
\sqrt{\frac{\Lambda}{n}}\left(\frac{\kappa_{0}}{\kappa_{\max }}\right) \leq \frac{1}{r} .
$$

If the equality is attained, we know that ${ }^{(n-1)} h_{i j}^{0}={ }^{(n-1)} \delta_{i j}$ and, moreover, we may also apply the equality case in Theorem 1. So Theorem 2 is proved.

\section{REFERENCES}

[1] W. Boucher, G. W. Gibbons, G. T. Horowitz, Uniqueness theorem for anti-de Sitter spacetime, Physical Review D, 30 (1984), 2447.

[2] P. T. Chruściel, Remarks on rigidity of the de Sitter metric, [homepage.univie.ac.at/piotr.chrusciel/papers/deSitter/deSitter2.pdf]. 
[3] M. Kanai, On a differential equation characterizing a Riemannian structure of a manifold, Tokyo J. Math., 6 (1983), 143.

[4] M. Obata, Certain conditions for a Riemannian manifold to be isometric to a sphere, J. Math. Soc. Japan, 14 (1962), 333.

[5] J. Lafontaine, A remark about static space times, J. Geom. Phys., 59 (2003), 50.

[6] J. P. Bourguignon, Une stratification de l'espace des structures riemanniennes, Comp. Math., 30 (1975), 1.

[7] A. E. Fischer, J. E. Marsden, Linearization stability of nonlinear partial differential equations, Proc. Symp. Pure Math., 27 (1975), 219.

[8] J. Qing, W. Yuan, A note on static spaces and related problems, J. Geom. Phys., bf 74 (2013), 18.

[9] Y. Shen, A note on Fischer-Marsden's conjecture, Proc. Amer. Math. Soc., 125 (1997), 901.

[10] R. C. Reilly, Applications of the Hessian operator in a Riemannian manifold, Indiana Univ. Math. J., 26 (1977), 459.

[11] O. Kobayashi, A differential equation arising from scalar curvature, J. Math. Soc. Japan, 34 (1982), 665.

[12] J. Lafontaine, Sur la géométrie d'une généralisation de l'équation différentielle d'Obata, J. Math. Pures Appl., 9 (1983), 63.

[13] L. Lindblom, Static uniform-density stars must be spherical in general relativity, J. Math. Phys., 29 (1988), 436.

[14] M. Anderson, On stationary vacuum solutions to the Einstein equations, Ann. Henri Poincaré, 1 (2005), 977.

[15] L. Andersson, M. Dahl, Scalar curvature rigidity for asymptotically locally hyperbolic manifolds, Ann. Glob. Anal. Geom., 16 (1998), 1.

[16] P. T. Chruściel, M. Herzlich, The mass of asymptotically hyperbolic Riemannian manifolds, Pacific J. Math., 212 (2003), 231.

[17] P. T. Chruściel, W. Simon, Towards the classification of static vacuum spacetimes with negative cosmological constant, J. Math. Phys., 42 (2001), 1779.

[18] G. J. Galloway, S. Surya, E. Woolgar, On the geometry and mass of static, asymptotically AdS spacetimes, and the uniqueness of the AdS soliton, Commun. Math. Phys., 241 (2003), 1.

[19] O. Hijazi, S. Montiel, Uniqueness of the AdS spacetime among static vacua with prescribed null infinity, Adv. Th. Math. Phys. (2014). [arxiv.org/abs/1211.5651].

[20] J. M. Lee, The spectrum of an asymptotically hyperbolic Einstein manifold, Comm. Anal. Geom., 2 (1995), 253.

[21] X. Wang, The mass of asymptotically hyperbolic manifolds, J. Diff. Geom., 57 (2001), 273.

[22] X. Wang, Uniqueness of the ADS space time in any dimension, Acta Math. Sin., 21 (2005), 917.

[23] M. Anderson, On the structure of solutions to the static vacuum Einstein equations, Ann. H. Poincaré, 1 (2000), 995.

[24] G. Bunting, A. K. M. Masood-ul-Alam, Nonexistence of multiple black holes in asymptotically euclidean static vacuum space-time, Gen. Rel. Grav., 19 (1987), 147. 
[25] P. T. Chruściel, The classification of static vacuum space-times containing an asymptotically flat spacelike hypersurface with compact interior, Class. Quantum Grav., 16 (1999), 661.

[26] W. Israel, Event horizons in static vacuum space-times, Phys. Rev. 164 (1967), 1776.

[27] G. W. Gibbons, S. A. Hartnoll, C. N. Pope, Bohm and Einstein-Sasaki metrics, black holes and cosmological event horizons, Physical Review D, 67 (2003), 84024.

[28] C. Böhm, Inhomogeneous Einstein metrics on low-dimensional spheres and other low-dimensional spaces, Invent. Math., 134 (1998), 145.

[29] P. G. LeFloch, L. Rozoy, Uniqueness of Kottler spacetime and the Besse conjecture, C. R. Acad. Sci. Paris, Ser. I, 348 (2010), 1129.

[30] A. K. M. Masood-ul-Alam, Static equations with positive cosmological constant, [msc.tsinghua.edu.cn/upload/news_201241144839.pdf].

[31] M. Herzlich, Extremality for the Vafa-Witten bound on the sphere, Geom. Funct. Anal., 15 (2005), 1153.

[32] C. Vafa, E. Witten, Eigenvalue inequalities for fermions in gauge theories, Commun. Math. Phys. 95 (1984), 257.

[33] J. Qing, On the rigidity for conformally compact Einstein manifolds, Intern. Math. Res. Not., 21 (2003), 1141.

[34] A. Besse, Einstein manifolds, Springer, New York,(1987).

[33] J. F. Escobar, Conformal deformation of a Riemannian metric to a scalar flat metric with constant mean curvature on the boundary, Ann. of Math., 136 (1992), 1.

[36] H. Baum, T. Friedrich, R. Grünewald, I. Kath, Twistor and Killing Spinors on Riemannian Manifolds, Teubner-Texte zur Mathematik, Band 124, TeubnerVerlag, Sttugart/Leipzig, 1991.

[37] T. Friedrich, Dirac Operators in Riemannian Geometry, AMS Graduate Studies in Math., vol. 25, 2000.

[38] H. B. Lawson, M. L. Michelsohn, Spin Geometry, Princeton Math. Series, vol. 38, Princeton University Press, 1989.

[39] J.-P. Bourguignon, O. Hijazi, J.-L. Milhorat, A. Moroianu, S. Moroianu, A Spinorial Approach to Riemannian and Conformal Geometry, to appear in Monographs in Mathematics, EMS, 2014.

[40] C. Bär, Extrinsic bounds of the Dirac operator, Ann. Glob. Anal. Geom., 16 (1998), 573.

[41] O. Hijazi, S. Montiel, X. Zhang, Dirac operator on embedded hypersurfaces, Math. Res. Lett, 8 (2001), 195.

[42] O. Hijazi, S. Montiel, X. Zhang, Conformal lower bounds for the Dirac operator of embedded hypersurfaces, Asian J. Math., 6 (2002), 23.

[43] N. Ginoux, The Dirac Spectrum, Lecture Notes in Mathematics, 1976 (2009).

[44] N. Hitchin, Harmonic spinors, Adv. in Math., 14 (1974), 1. 
(Hijazi) Institut Élie Cartan de Lorraine, Université de Lorraine, Nancy, B.P. 239, 54506 Vandeeuvre-Lès-Nancy Cedex, France

E-mail address: Oussama.Hijazi@univ-lorraine.fr

(Montiel) Departamento de Geometría y Topología, Universidad de Granada, 18071 Granada, Spain

E-mail address: smontiel@ugr.es

(Raulot) Laboratoire de Mathématiques R. Salem UMR 6085 CNRSUniversité de Rouen Avenue de L'Université, BP.12 Technopôle DU Madrillet 76801 Saint-Étienne-Du-Rouvray, France.

E-mail address: simon.raulot@univ-rouen.fr 